\title{
Evaluación de calidad de la atención prenatal en centros de salud de la provincia de Lambayeque adscritos a la Gerencia Regional de Salud Lambayeque
}

\author{
Percy Díaz -Morón ${ }^{1, a}$
}

\section{RESUMEN}

Objetivo. Evaluar la calidad de la atención prenatal en centros de salud de la provincia de Lambayeque, durante enero a marzo de 2019. Materiales y métodos: Se evaluaron 325 usuarias y 51 profesionales de la salud, procedentes de los centros de salud de la provincia de Lambayeque adscritos a la Gerencia Regional de Salud; se recolectó información mediante instrumentos como hoja de verificación, registro de datos del carné perinatal y encuestas; se evaluó las dimensiones de calidad: competencia, seguridad, accesibilidad, satisfacción y comodidades, las que fueron dicotomizadas como adecuado e inadecuado y luego analizadas mediante la estadística descriptiva. Resultados: Se encontró que las usuarias percibieron en $79,1 \%$ la capacidad profesional adecuada, en la seguridad $76,9 \%$ indicó preferencia por quien la atiende, $60 \%$ consideró adecuada la documentación requerida, $65 \%$ entienden las explicaciones, 57,5 \% percibió como inadecuada la calidad de la atención y el $64,3 \%$ percibió que la comodidad es adecuada. El $80,4 \%$ de profesionales conoce las guías de atención prenatal, 76,4 \% las usa, 60,7 \% considera que la calidad de su aplicación es adecuada, en la seguridad 76,5\% consideró que la ayuda del auxiliar es adecuada, 96,1\% cuenta con guías de atención, 49,1 \% está satisfecho en su centro laboral, y el $76,5 \%$ percibió adecuada la comodidad del consultorio. Conclusión: Se concluye que en la evaluación de calidad de la atención prenatal, las dimensiones competencia y seguridad percibidas por usuarias y profesionales fueron adecuadas, y la competencia profesional para el registro del carné perinatal fue inadecuado. Asimismo, la seguridad percibida por usuarias y profesionales fue adecuada, pero la accesibilidad fue inadecuada para usuarias y adecuada para profesionales. Y en relación a la comodidad esta fue percibida como adecuada para ambos grupos de participantes en el estudio.

Palabras Clave: Salud. Calidad de la Atención Médica. Atención Prenatal.

\footnotetext{
${ }^{1}$ Universidad Señor de Sipán. Escuela de Medicina Humana. Hospital Regional Lambayeque. Lambayeque, Perú.

a Magíster en Ciencias. Médico Especialista en Medicina Familiar y Comunitaria.
} 


\section{Quality assessment of prenatal care in health centers of the province of Lambayeque attached to the Regional Health Management Lambayeque}

\section{ABSTRACT}

Objective. Assess the quality of prenatal care in health facilities in Lambayeque province, from January to March 2019. Materials and methods: 325 users and 51 health professionals were evaluated from health centers in the province of Lambayeque attached to the Regional Health Management, collecting information through instruments such as verification sheets, data record of the perinatal card and surveys; evaluating the quality dimensions: competence, safety, accessibility, satisfaction and comforts, which were dichotomized as adequate and inadequate and then analyzed using descriptive statistics. Results: Users were found to have received adequate professional capacity at $79.1 \%$, in safety $76.9 \%$ indicated preference for the caregiver, $60 \%$ considered the required documentation appropriate, $65 \%$ understand the explanations, $57.5 \%$ perceived as inadequate the quality of care and $64.3 \%$ perceived that comfort is adequate. $80.4 \%$ of professionals know the prenatal care guidelines, $76.4 \%$ use them, $60.7 \%$ believe that the quality of their application is adequate, regarding safety $76.5 \%$ considered that the auxiliary's aid is adequate, $96.1 \%$ has care guides, $49.1 \%$ is satisfied in their workplace, and $76.5 \%$ perceived the comfort of the office as adequate. Conclusion: It is concluded that in the quality assessment of prenatal care, the competitive and safety dimensions, perceived by users and professionals were adequate, and professional competence for perinatal card registration was inadequate. Likewise, the security perceived by users and professionals was adequate but accessibility was inadequate for users and suitable for professionals. And, in relation to comfort this was perceived as suitable for both.

Keywords: Health. Quality of Health Care. Prenatal care.

\section{INTRODUCCIÓN}

En nuestro país, la calidad es un principio rector de la política de salud como un derecho ciudadano en donde el eje de la atención es la satisfacción de los usuarios centrada en sus dimensiones técnico-científicas, humanas y del entorno cuyos atributos la competencia profesional, eficacia, eficiencia, continuidad, seguridad, accesibilidad, satisfacción del usuario, y comodidad de la atención son fundamentales para el logro de objetivos (1).

En el mundo, en los países en situación de pobreza como el nuestro, es característica la detección tardía del embarazo y deficientes atenciones prenatales, conllevando a que desde el momento de la gestación los niños presenten ya desigualdades y las gestantes expuestas a riesgos que podrían llegar a resultados negativos como mayor morbimortalidad ${ }^{(1)}$.

A nivel de América Latina, en los últimos 20 años ha disminuido en $50 \%$ la mortalidad materna, de 16,000 a 7,300 muertes. Sin embargo, nuestro país que se encuentra entre los cinco países que más ha progresado en términos de reducción de mortalidad materna, de 185 a 68 muertes maternas por cada 100,000 nacidos vivos (2000 -2015), aun así, sigue ocupando el décimo lugar entre los países con la tasa más alta de mortalidad materna en América Latina, ubicándose por encima del promedio latinoamericano, según datos del Programa de Naciones Unidas para el Desarrollo ${ }^{(2,3,4)}$.
Las barreras para la atención prenatal adecuada como la falta de competencias profesionales del equipo de salud, problemas en la organización, inaccesibilidad geográfica, barreas culturales, desconfianza de las mujeres en los sistemas de salud, falta de promoción en la comunidad y las familias, la cobertura parcial del seguro integral de salud, no contribuyen a que esta sea de calidad y, por ende, la persistencia de la mortalidad materno perinatal (5).

La inadecuada calidad de atención prenatal en los establecimientos de salud de nuestro país es uno de los mayores obstáculos para enfrentar los problemas sanitarios materno perinatales con éxito ${ }^{(5,6)}$. En su mayoría los factores de riesgo para la salud de la madre se identifican durante la atención prenatal, muchos son prevenibles o modificables por medio de la supresión del riesgo o por acciones diagnósticas o terapéuticas oportunas que favorecerán resultados deseables en la salud materno infantil, actualmente la mayoría de profesionales han llegado a aceptar que la única forma de mejorar las condiciones relativas al parto es mediante una atención prenatal oportuna y de calidad (5,6).

Aunque la cobertura de la atención prenatal en el Perú ha aumentado en los últimos años, es frecuente la captación tardía en el último trimestre del embarazo, con bajo promedio de atenciones prenatales y el llenado incompleto de la historia clínica que se ha convertido en una desafortunada costumbre en la práctica médica moderna; por ejemplo, en 
la mayoría de los hospitales del Minsa donde las gestantes ingresan para su atención de parto se encontró deficiencia en el llenado del carné perinatal por los profesionales que realizan la atención prenatal, dificultando la identificación y prevención de posibles complicaciones ${ }^{(7)}$. Los resultados de esta investigación, serán de utilidad en los procesos de toma de decisiones de las jefaturas de los centros de salud de la provincia de Lambayeque adscritos a la Gerencia Regional de Salud, y les permitirá implementar acciones estratégicas, para mejorar la calidad de la atención prenatal.

\section{METODOLOGÍA}

El tipo de estudio fue descriptivo prospectivo, el tamaño de la muestra se calculó considerando todos los centros de salud de la provincia de Lambayeque adscritos a la Gerencia Regional de Salud. Para cada establecimiento, se computó con la fórmula de un muestreo estratificado para variables cualitativas y con población conocida. La muestra ajustada, para las usuarias fue de 325 , la cual se distribuyó por afijación proporcional en estratos, que fueron los consultorios de Medicina General, Enfermería, Obstetricia, Psicología y Odontología. Así mismo la muestra para los profesionales médicos y obstetras que brindaron la atención prenatal fue de 51 para los 13 centros de salud. Se utilizó en una primera etapa hojas de registro y hojas de verificación de datos, donde se recolectó información de los carnés materno perinatales y las historias clínicas de atención prenatal realizados en los últimos dos años en todos los centros de salud de la provincia de Lambayeque adscritos a la Gerencia Regional de Salud.

Así mismo, se evaluó la relación de las actividades propuestas para la atención prenatal consignadas en el carné perinatal. Se clasificó como Bueno, aquel registro que se encuentre bien llenado, de acuerdo a las normas establecidas; Enmendaduras, aquel registro que se encuentre con borrones o algún tipo de arreglo que invalide al dato por considerarlo no legible, pero consignados en el lugar adecuado; No registrado, cuando el ítem estudiado no figure en la casilla que le corresponda por falta de llenado; y Registro inadecuado, aquel registro que consigne otra información no establecida en los manuales. Luego para procesar los datos se empleó la siguiente dicotomía: Adecuado (Bueno) e Inadecuado (Enmendaduras, No registrado y Registro inadecuado). Todas estas actividades están consignadas en los manuales de normas y procedimientos del Ministerio de Salud, y en el Manual del Comité Latinoamericano de Atención Prenatal (CLAP) en sus publicaciones científicas, adoptadas por el Perú desde 1983 y trasladadas a los esquemas del Ministerio de Salud ${ }^{(8,9)}$.

Las hojas de registro fueron estandarizadas en ocho ítems, distribuidas en las dimensiones competencia profesional, eficacia eficiencia seguridad, continuidad, accesibilidad, satisfacción al usuario y comodidades. Asimismo, en una segunda etapa se aplicó un cuestionario a las usuarias y un cuestionario a los profesionales que brindaron la atención prenatal. Los cuestionarios fueron validados por juicio de expertos; se exploró la validez de contenido y se demostró que los cuestionarios poseen la confiabilidad requerida para su uso $(\alpha=, 853)$.

El cuestionario para las ususarias fue de 22 preguntas distribuidas en las dimensiones seguridad, continuidad, accesibilidad, satisfacción al usuario y comodidades. Y el cuestionario para los profesionales fue de 33 preguntas distribuidas en las dimensiones competencia profesional, seguridad, continuidad, accesibilidad, satisfacción al usuario y comodidades. No se afectó ninguna norma de privacidad, derechos legales y derechos humanos, ya que en la revisión de los carnés materno perinatales se conservó el anonimato de los datos de las pacientes, no atentando contra la ética en los casos sometidos a este estudio, pues aún cuando las pacientes desconocían su participación, la revisión de sus datos fue considerada en forma casuística y no personalizada.

Los datos de los instrumentos aplicados se digitaron en SSPS versión 2.2, se analizó a través de estadísticas descriptivas de la variables en estudio.

\section{RESULTADOS}

En los cuestionarios aplicados, en la dimensión referida a la competencia profesional percibida por las usuarias, se observó que en el 79,1 \% la capacidad del profesional es adecuada, en $66,4 \%$ el tratamiento con medicamentos es adecuado y en 78,2 \% la información brindada es adecuada. Por otro lado, en relación a la competencia profesional percibida por los profesionales, se observó que el 80,4 \% conoce las guías de atención prenatal y el 76,4 \% hace uso de ellas. Así mismo, 60,7\% considera que la calidad de aplicación de estas normas es adecuada; el 84,3\% percibe el registro de la historia clínica perinatal básica y el carné perinatal como adecuado, así como el 70,6 \% considera que las estrategias de mejora del control prenatal son adecuadas. También se observó que el 52,9 \% de profesionales que realizan atención prenatal contaba con otros estudios e indicaban que la calidad de aplicación del flujograma de atención (60,7 \%) es adecuado. Sin embargo, también se encontró que tanto la calidad de los recursos asignados para el control prenatal $(39,2 \%)$ y los equipos que tienen para la realización de la atención prenatal son inadecuados (Tabla 1).

Tabla 1. Evaluación de la competencia profesional en la atención prenatal en los centros de salud de la provincia de Lambayeque según percepciones de usuarias y profesionales. 


\begin{tabular}{|c|c|c|}
\hline Ítems evaluados por profesionales & $\mathbf{n}$ & $\%$ \\
\hline \multicolumn{3}{|l|}{ Conocimiento de guías atención } \\
\hline Sí & 41 & 80,4 \\
\hline No & 10 & 19,6 \\
\hline \multicolumn{3}{|l|}{ Uso de Guías Atención } \\
\hline Sí & 39 & 76,4 \\
\hline No & 12 & 23,6 \\
\hline \multicolumn{3}{|c|}{ Calidad de aplicación normas y guías de atención } \\
\hline Adecuado & 31 & 60,7 \\
\hline Inadecuado & 20 & 39,3 \\
\hline \multicolumn{3}{|c|}{ Calidad del registro dela historia clínica y carné perinatal } \\
\hline Adecuado & 43 & 84,3 \\
\hline Inadecuado & 8 & 15,7 \\
\hline \multicolumn{3}{|c|}{ Pregunta sobre hábitos alimenticios y nutrición } \\
\hline Sí & 49 & 96,1 \\
\hline No & 2 & 3,9 \\
\hline \multicolumn{3}{|l|}{ Cuenta con otro tipo de estudios } \\
\hline Sí & 27 & 52,9 \\
\hline No & 24 & 47,1 \\
\hline \multicolumn{3}{|c|}{ Calidad del reparto de responsabilidades } \\
\hline Adecuado & 42 & 82,4 \\
\hline Inadecuado & 9 & 17,6 \\
\hline \multicolumn{3}{|c|}{ Calidad de estrategias mejora de la atención prenatal } \\
\hline Adecuado & 36 & 70,6 \\
\hline Inadecuado & 15 & 29,4 \\
\hline \multicolumn{3}{|c|}{ Calidad de supervisión de atención prenatal } \\
\hline Adecuado & 22 & 43,1 \\
\hline Inadecuado & 29 & 56,9 \\
\hline \multicolumn{3}{|c|}{ Calidad del Flujograma de Atención Prenatal } \\
\hline Adecuado & 31 & 60,7 \\
\hline Inadecuado & 20 & 39,3 \\
\hline \multicolumn{3}{|c|}{ Calidad de recursos para atención prenatal } \\
\hline Adecuado & 20 & 39,2 \\
\hline Inadecuado & 31 & 60,8 \\
\hline \multicolumn{3}{|c|}{ Calidad de equipos para atención prenatal } \\
\hline Adecuado & 15 & 29,4 \\
\hline Inadecuado & 36 & 70,6 \\
\hline
\end{tabular}

Fuente: Cuestionario a Usuarias y Profesionales de Salud 
Por otro lado, en cuanto a la dimensión competencia profesional, evaluada según el registro completo del carné perinatal por los profesionales, se evidenció que la evaluación del peso materno y crecimiento fetal fueron registrados inadecuadamente en $86,2 \%$, así como los antecedentes obstétricos y vacunación antitetánica fueron registrados inadecuadamente en $82 \%$ y $72 \%$ respectivamente, no siendo así la toma de presión arterial, examen clínico y ginecobstétrico y la presentación fetal cuyos registros $90,5 \%, 78 \%$ y $82,2 \%$ respectivamente fueron adecuados (Tabla 2 )

Tabla 2. Evaluación de la competencia profesional en la atención prenatal, según registro del carné perinatal por profesionales que atienden en centros de salud de la provincia de Lambayeque.

\begin{tabular}{|c|c|c|}
\hline Ítems Evaluados del carné perinatal & $\mathbf{n}$ & $\%$ \\
\hline \multicolumn{3}{|l|}{ Registro de toma de presión arterial } \\
\hline Adecuado & 294 & 90,5 \\
\hline Inadecuado & 31 & 9,5 \\
\hline \multicolumn{3}{|c|}{ Registro de evaluación peso materno y crecimiento fetal } \\
\hline Adecuado & 45 & 13,8 \\
\hline Inadecuado & 280 & 86,2 \\
\hline \multicolumn{3}{|c|}{ Registro de determinación edad de riesgo ( $<15$ y >35 años) } \\
\hline Adecuado & 133 & 40,9 \\
\hline Inadecuado & 192 & 59,1 \\
\hline \multicolumn{3}{|l|}{ Registro de Antecedentes Obstétricos } \\
\hline Adecuado & 58 & 17,8 \\
\hline Inadecuado & 267 & 82,2 \\
\hline \multicolumn{3}{|l|}{ Registro de vacunación antitetánica } \\
\hline Adecuado & 87 & 26,8 \\
\hline Inadecuado & 238 & 73,2 \\
\hline \multicolumn{3}{|l|}{ Registro de batería análisis gestante } \\
\hline Adecuado & 134 & 41,2 \\
\hline Inadecuado & 191 & 58,7 \\
\hline \multicolumn{3}{|c|}{ Registro de examen clínico y gineco obstétrico } \\
\hline Adecuado & 255 & 78,5 \\
\hline Inadecuado & 70 & 21,5 \\
\hline \multicolumn{3}{|l|}{ Registro de papanicolaou } \\
\hline Adecuado & 19 & 5,8 \\
\hline Inadecuado & 306 & 94,2 \\
\hline \multicolumn{3}{|l|}{ Registro de presentación fetal } \\
\hline Adecuado & 267 & 82,2 \\
\hline Inadecuado & 58 & 17,8 \\
\hline
\end{tabular}

Fuente: Registro del Carne Perinatal por Profesionales 
Así mismo, en la dimensión seguridad se encontró que el 76,9 \% de las usuarias indicó preferencia por su profesional que la atiende en la atención prenatal, además un 96 \% indicó que recibe explicación de la importancia de la atención prenatal, 89,5\% valora la información que le dan en la atención prenatal, así como también el 89,2 \% indican que recibieron recomendaciones en la atención prenatal. Además, en relación a la dimensión seguridad se observó que el $76,5 \%$ de los profesionales consideró que la ayuda del personal auxiliar es adecuada y $80,4 \%$ consideró que la calidad de los resultados del laboratorio es adecuada (Tabla 3).

Tabla 3. Evaluación de la seguridad en la atención prenatal en los centros de salud de la provincia de Lambayeque según percepciones de usuarias y profesionales

\begin{tabular}{|c|c|c|}
\hline Ítems evaluados por usuarias & $\bar{n}$ & $\%$ \\
\hline \multicolumn{3}{|l|}{ Preferencia por el mismo profesional } \\
\hline Sí & 250 & 76,9 \\
\hline No & 75 & 23,1 \\
\hline \multicolumn{3}{|l|}{ Explicación de importancia de la atención prenatal } \\
\hline Sí & 308 & 96,0 \\
\hline No & 13 & 4,0 \\
\hline \multicolumn{3}{|l|}{ Explicación del motivo de las recomendaciones } \\
\hline Sí & 290 & 89,2 \\
\hline No & 35 & 10,8 \\
\hline \multicolumn{3}{|l|}{ Valoración de la información brindada } \\
\hline Sí & 291 & 89,5 \\
\hline 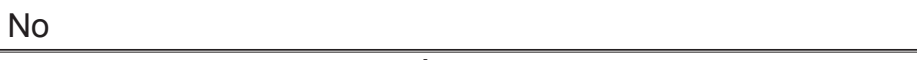 & 34 & 10,5 \\
\hline Ítems evaluados por profesionales & $\mathrm{n}$ & $\%$ \\
\hline \multicolumn{3}{|l|}{ Calidad de ayuda del personal auxiliar } \\
\hline Adecuado & 39 & 76,5 \\
\hline Inadecuado & 12 & 23,5 \\
\hline \multicolumn{3}{|l|}{ Calidad de resultados de laboratorio } \\
\hline Adecuado & 41 & 80,4 \\
\hline Inadecuado & 10 & 19,6 \\
\hline
\end{tabular}

Fuente: Cuestionario a Usuarias y Profesionales de Salud

En cuanto a la dimensión accesibilidad, se observó que el $60 \%$ de las usuarias consideró que la documentación requerida para su atención prenatal era adecuada, $65 \%$ entienden las explicaciones que su profesional les brinda y solo el $27,7 \%$ consideró el horario de atención como adecuado. Así mismo, en dimensión accesibilidad percibida por los profesionales se encontró que el 96,1 \% cuenta con normas y guías de atención prenatal, $65 \%$ manifestaron que sí entienden a las usuarias a las que les brindan atención prenatal y 56,9 \% percibieron como adecuada la distancia que recorren las usuarias para llegar al centro de salud. También se encontró que la percepción del horario de atención del consultorio $(94,1 \%)$ y el tiempo de espera para la atención prenatal $(68,6 \%)$ son adecuados (tabla 4$)$. 
Tabla 4. Evaluación de la accesibilidad en la atención prenatal en los centros de salud de la provincia de Lambayeque según percepciones de usuarias y profesionales.

\begin{tabular}{|c|c|c|}
\hline Ítems evaluados por usuarias & $\mathbf{n}$ & $\%$ \\
\hline \multicolumn{3}{|l|}{ Documentación requerida en la atención prenatal } \\
\hline Adecuado & 195 & 60,0 \\
\hline Inadecuado & 130 & 40,0 \\
\hline \multicolumn{3}{|l|}{ Horario de la atención prenatal } \\
\hline Adecuado & 90 & 27,7 \\
\hline Inadecuado & 235 & 72,3 \\
\hline \multicolumn{3}{|l|}{ Tiempo de espera para atención prenatal } \\
\hline Adecuado & 141 & 43,4 \\
\hline Inadecuado & 184 & 56,6 \\
\hline \multicolumn{3}{|l|}{ Precepción de la distancia para llegar al centro de salud } \\
\hline Adecuado & 207 & 63,7 \\
\hline Inadecuado & 118 & 36,3 \\
\hline \multicolumn{3}{|l|}{ Entendimiento de las explicaciones del profesional } \\
\hline Sí & 211 & 65,0 \\
\hline e & 114 & 35,0 \\
\hline Ítems evaluados por profesionales & $\mathrm{n}$ & $\%$ \\
\hline \multicolumn{3}{|l|}{ Cuenta con normas y guías de atención prenatal } \\
\hline Sí & | & 96,1 \\
\hline No & 2 & 3,9 \\
\hline \multicolumn{3}{|l|}{ Horario de la atención prenatal } \\
\hline Adecuado & 48 & 94,1 \\
\hline Inadecuado & 3 & 5,9 \\
\hline \multicolumn{3}{|l|}{ Tiempo de espera para atención prenatal } \\
\hline Adecuado & 35 & 68,6 \\
\hline Inadecuado & 16 & 31,4 \\
\hline \multicolumn{3}{|l|}{ Precepción de la distancia para llegar al centro de salud } \\
\hline Adecuado & 29 & 56,9 \\
\hline Inadecuado & 22 & 43,1 \\
\hline \multicolumn{3}{|l|}{ Entendimiento de las explicaciones a la usuaria } \\
\hline Sí & 47 & 92,2 \\
\hline No & 4 & 7,8 \\
\hline
\end{tabular}

Fuente: Cuestionario a Usuarias y Profesionales de Salud

En relación a la dimensión satisfacción se observó que el 51,1 \% de las usuarias manifiesta no se escuchada por su profesional y el 51,4 \% percibió como adecuado la forma cómo le responde. Sin embargo, el 50,2 \% de ellas no percibió cambios en su estado de salud tras las consultas prenatales y el 57,5\% percibió como inadecuada la calidad de la atención prenatal recibida. Así mismo, en la misma dimensión el 49,1 \% de los profesionales indicó estar satisfecho en su centro laboral, el 54,9\% percibió como adecuada la remuneración que recibe, el 45,1 \% recibió información de su desempeño en la atención prenatal, el 78,4 \% reveló que el trato recibido en su ambiente de trabajo es adecuado y el 72,5\% indicó que es adecuada la organización de su servicio de atención prenatal. Sin embargo, el 41,2 \% percibe que la institución no le proporciona los recursos para la atención prenatal, además revelan que no reciben capacitaciones ni reconocimientos por la atención prenatal que realizan en $64,7 \%$ y $70,6 \%$ respectivamente (Tabla 5). 
Tabla 5. Evaluación de la satisfacción en la atención prenatal en los centros de salud de la provincia de lambayeque según percepciones de usuarias y profesionales.

\begin{tabular}{|c|c|c|}
\hline $\begin{array}{l}\text { Ítems evaluados por usuarias } \\
\end{array}$ & $\mathbf{n}$ & $\%$ \\
\hline \multicolumn{3}{|l|}{ Percibió mejoras luego de su atención prenatal } \\
\hline Sí & 162 & 49,8 \\
\hline No & 163 & 50,2 \\
\hline \multicolumn{3}{|l|}{ El profesional la escucha durante la atención prenatal } \\
\hline Sí & 159 & 48,9 \\
\hline No & 166 & 51,1 \\
\hline \multicolumn{3}{|l|}{ Molestias del profesional al ser preguntado } \\
\hline Sí & 41 & 12,6 \\
\hline No & 284 & 87,4 \\
\hline \multicolumn{3}{|l|}{ Forma como le responde el profesional } \\
\hline Adecuado & 167 & 51,4 \\
\hline Inadecuado & 158 & 48,6 \\
\hline \multicolumn{3}{|l|}{ Percepción de la calidad de la atención prenatal } \\
\hline Adecuado & 138 & 42,5 \\
\hline Inadecuado & 187 & 57,5 \\
\hline Items evaluados por profesionales & $\mathbf{n}$ & $\%$ \\
\hline \multicolumn{3}{|l|}{ Organización del servicio de atención prenatal } \\
\hline Adecuado & 37 & 72,5 \\
\hline Inadecuado & 14 & 27,5 \\
\hline \multicolumn{3}{|l|}{ Recibe Información de su Desempeño en Atención Prenatal } \\
\hline Sí & 23 & 45,1 \\
\hline No & 28 & 54,9 \\
\hline \multicolumn{3}{|l|}{ Institución Proporciona Recursos para Atención Prenatal } \\
\hline Sí & 21 & 41,2 \\
\hline No & 30 & 58,8 \\
\hline \multicolumn{3}{|l|}{ Recibe Reconocimientos por Logros Atención Prenatal } \\
\hline Sí & 15 & 29,4 \\
\hline No & 36 & 70,6 \\
\hline \multicolumn{3}{|l|}{ Institución Proporciona Capacitación en Atención Prenatal } \\
\hline Sí & 18 & 35,3 \\
\hline No & 33 & 64,7 \\
\hline \multicolumn{3}{|l|}{ Remuneración de acuerdo a la función } \\
\hline Adecuado & 28 & 54,9 \\
\hline Inadecuado & 23 & 45,1 \\
\hline \multicolumn{3}{|l|}{ Trato recibido en ambiente de trabajo } \\
\hline Adecuado & 40 & 78,4 \\
\hline Inadecuado & 11 & 21,6 \\
\hline \multicolumn{3}{|l|}{ Satisfacción en su Centro Laboral } \\
\hline Sí & 25 & 49,1 \\
\hline No & 26 & 50,9 \\
\hline
\end{tabular}

Fuente: Cuestionario a usuarias y profesionales de salud. 
En relación a la dimensión comodidad percibida por las usuarias, se observó que el 63,7 \% de estas consideró la ubicación del consultorio de atención prenatal como adecuado, un 64,3\% percibió que la comodidad del consultorio es adecuado, así como también un $60 \%$ consideró que los ambientes del consultorio prenatal son adecuados. En cuanto a la comodidad percibida por los profesionales, se encontró que el 58,8 \% consideró la ubicación del consultorio de atención prenatal como adecuado, el 76,5 $\%$ percibió que la comodidad del consultorio es adecuado, el 50,1 \% consideró que los ambientes del consultorio prenatal son adecuados y un 70,6 \% consideró adecuada la duración de la consulta (Tabla 6)

Tabla 6. Evaluación de la comodidad en la atención prenatal en los centros de salud de la provincia de Lambayeque según percepciones de usuarias y profesionales.

\begin{tabular}{|c|c|c|c|c|}
\hline \multirow{3}{*}{ Ítems evaluados } & \multicolumn{4}{|c|}{ Percepciones } \\
\hline & \multicolumn{2}{|c|}{ Usuarias } & \multicolumn{2}{|c|}{ Profesionales } \\
\hline & $\mathrm{n}$ & $\%$ & $\mathrm{n}$ & $\%$ \\
\hline \multicolumn{5}{|c|}{ Ubicación del consultorio de atención prenatal } \\
\hline Adecuado & 207 & 63,7 & 30 & 58.8 \\
\hline Inadecuado & 118 & 36,3 & 21 & 41.2 \\
\hline \multicolumn{5}{|c|}{ Comodidad del consultorio de atención prenatal } \\
\hline Adecuado & 210 & 64,6 & 39 & 76.5 \\
\hline Inadecuado & 115 & 35,4 & 12 & 23.5 \\
\hline \multicolumn{5}{|c|}{ Ambiente del consultorio de atención prenatal } \\
\hline Adecuado & 195 & 60,0 & 26 & 50.1 \\
\hline Inadecuado & 130 & 40,0 & 25 & 49.9 \\
\hline \multicolumn{5}{|l|}{ Tiempo de consulta atención prenatal } \\
\hline Adecuado & 227 & 69,8 & 36 & 70.6 \\
\hline Inadecuado & 98 & 30,2 & 15 & 29.4 \\
\hline
\end{tabular}

Fuente: Cuestionario a usuarias de servicios de atención prenatal

\section{DISCUSION}

En relación a la dimensión competencia profesional, la percepción de las usuarias y los profesionales sobre la atención prenatal fue adecuada, resultados similares a los que obtuvo Flores et al. en el 2016 en su estudio, donde las usuarias se sentían satisfechas y los usuarios internos regularmente satisfechos ${ }^{(10)}$.

La competencia profesional evaluada según el registro del carné perinatal por los profesionales, ningún registro fue adecuado, similar al estudio de Alfaro et al. en el 2012, quienes encontraron que existe una baja cobertura de atención prenatal con cumplimiento de criterios de calidad, pues la información obtenida del carné perinatal registradas en el Sistema Informático Perinatal (SIP 2000) durante las consultas de control prenatal es incompleta y errónea(09). Así mismo para que el registro de análisis de laboratorio, el peso materno y crecimiento fetal sea adecuado se requiere ocho atenciones y seis para el examen clínico y gineco obstétrico. Por tanto, podemos determinar que el registro de la mayoría de actividades de la atención prenatal son inadecuadas y no cumplen con lo estandarizado por la OMS ${ }^{(1,8)}$.

En la dimensión seguridad, las usuarias mostraron preferencia por la atención con en el mismo profesional y percibieron como adecuadas las explicaciones e información brindada en su atención prenatal; los profesionales que brindaron la atención prenatal percibieron como adecuada la calidad de los resultados de los análisis de laboratorio y ayuda del personal auxiliar, similar a los estudios de Larrea F. en el 2018, sobre percepción de la calidad de la atención prenatal en el centro de salud el Bosque Chiclayo donde la dimensión seguridad se consideró como buena (86\%) (11).

Respecto a la dimensión accesibilidad, en el estudio de Preciado et al. en el 2016, se encontró que las usuarias manifestaron estar regularmente satisfechas en $60,5 \%$, sin embargo, según el resultado encontrado en nuestro estudio la accesibilidad es adecuada, no coincidente con lo afirmado por el autor en mención, lo cual estaría relacionado a la menor distancia de las usuarias para llegar a los centros de salud ${ }^{(12)}$. Así mismo, la dimensión accesibilidad percibida por los profesionales en el estudio de Rodríguez H. en el 2015, sobre calidad de atención de salud de usuarios internos y externos encontró que el 80 $\%$ de usuarios internos consideró que las acciones y las actitudes adoptadas no facilitan los recursos necesarios para desarrollar sus capacidades y actividades, siendo percibido como inadecuado. Resultados que difieren con 
lo encontrado en nuestro estudio en el cual la accesibilidad fue percibida como adecuada por los profesionales ${ }^{(13)}$.

Así mismo, en la dimensión satisfacción, las usuarias no percibieron mejoras o cambios en su salud luego de la atención prenatal (forma de responder, capacidad de escucha y calidad de atención del profesional), resultados similares a los estudios de Camarena et al. en el 2007 sobre perspectiva de la calidad de atención prenatal en Chihuahua México y Munares OF. en el 2005, estudio sobre calidad del control prenatal en establecimientos del primer nivel de atención Lima, los cuales encontraron que la satisfacción de la usuaria es inadecuada pues se requiere "Orientación plena en cuanto a los cuidados" y un trato que vislumbre respeto e interés de los profesionales por sus emociones y sentimientos ${ }^{(14,15)}$.

Finalmente, en los resultados de la dimensión comodidades de la atención prenatal, en el estudio de Noguera et al. en el 2011 sobre auditoría del proceso de atención prenatal, encontró que las usuarias de atención prenatal están satisfechas con las condiciones de comodidad $93 \%$, y el estudio de Delgado R et al. en el 2003, sobre satisfacción del usuario interno que encontró que las variales que se asociaron a mayor confort era la percepción de adecuado mantenimiento de la infraestructura del establecimiento de salud. Estos resultados son similares a los encontrados en este estudio donde la comodidad del consultorio de atención prenatal fueron percibidos como adecuada por usuarias y profesionales ${ }^{(17,18)}$.

\section{CONCLUSIONES}

En la evaluación de calidad de la atención prenatal, se encontró que la dimensión competencia profesional y seguridad, percibida por usuarias y profesionales fue adecuada, y la competencia profesional para el registro de carné perinatal fue inadecuado; la dimensión seguridad percibida por usuarias y profesionales fue adecuada; la dimensión acesibilidad para usuarias y para los profesionales fue adecuada. Y en relación a la dimensión comodidad fue percibida como adecuada por las usuarias y profesionales.

Conflictos de interés: El autor declara que no existe conflicto de interés.

\section{Fuentes de financiamiento: Autofinanciado}

\section{REFERENCIAS BIBLIOGRÁFICAS}

1. Resolución Ministerial N¹42-2007/MINSA. Documento Técnico: «Estándares e indicadores de calidad en la atención Materno y Perinatal en los establecimientos que cumplen con funciones obstétricas y neonatales». Lima Ministerio de Salud del Perú; 2007.

2. Encuesta Demográfica de Salud Familiar (ENDES). Indicadores de Resultados de los Programas Presupuestales, Primer Semestre 2018. Lima. Instituto Nacional de Estadística e Informática; 2019. Disponible en: https:// proyectos.inei.gob.pe/endes/ppr.asp

3. Agenda la 2030, Objetivos del Desarrollo Sostenible. Objetivos del Desarrollo Sostenible al 2030. Washington, D.C: ONU; 2017. Disponible en: https://www.un.org/sustainabledevelopment/es/objetivos-de-desarrollo-sos- tenible/

4. Ministerio de Salud. Perú: Género, Salud y Desarrollo. Indicadores Básicos, 2018. Lima. MINSA; 2019. Disponible en: http://bvs.minsa.gob.pe/ local/

5. Seguro Integral de Salud. Estadísticas por Regiones y unidades ejecutoras 2010 - 2015. Lima. Ministerio de Salud; 2017. Disponible en: http:// www. sis.gob.pe/estad_indic_070723_2.htm

6. Recomendaciones de la OMS sobre atención prenatal para una experiencia positiva del embarazo. Washington, D.C.: Organización Panamericana de la Salud; 2018. Licencia: CC BY-NC-SA 3.0 IGO. Disponible en: https://iris.paho.org/bitstream/handle/10665.2/49550/9789275320334_spa. pdf?ua=1

7. Informe de ADJUNTIA N01-2017-DP-ADM. Derecho a una Maternidad Segura: Seguimiento de las Recomendaciones Defensoriales a Establecimientos de Salud. Lima. Defensoría del Pueblo; 2017. Disponible en: https://www.defensoria.gob.pe/modules/Downloads/informes/varios/2017/ Informe-de-Adjuntia-N-001-2017-DP-ADM_Derecho-a-la-Salud-Materna-.pdf

8. Resolución Ministerial N668-2014/MINSA. Guía Nacional de Atención Integral de Salud Sexual y Reproductiva. Lima. Ministerio de Salud; 2004. Disponible en: http://bvs.minsa.gob.pe/local/dgsp/63_guiasnac.pdf

9. Organización Panamericana de la Salud. Sistema Informático Perinatal, Historia Clínica Perinatal. Publicación CLAP/SMR N 1563. Washington, D.C: OPS,2007.https://www.paho.org/clap/index.php?option=com_docman\&view=download\&alias=190-clap-1564\&category_slug=ediciones-del-clap\&ltemid=219\&lang=es

10. Flores L, Molina J. Satisfacción en la calidad de Atención Prenatal según opinión de los usuarios y usurarias del Centro de Salud Solingalpa del Municipio de Matagalpa durante el Il semestre del año 2016. [Tesis]. Matagalpa-Nicaragua: Universidad Nacional Autónoma de Nicaragua, Managua; 2016. Disponible en: http://repositorio.unan.edu.ni/4524/

11. Larrea F, Harold R. Precepción sobre la calidad de atención prenatal que tienen las gestantes que acuden al centro de salud El Bosque, Octubre diciembre 2018. [Tesis]. Lambayeque-Perú: Universidad Nacional Pedro Ruiz Gallo; 2019. Disponible en:

12. Preciado F, et al. Satisfacción de las usuarias del consultorio materno del Puesto de Salud I-2 "Gerardo Gonzáles Villegas" Tumbes 2016. [Tesis]. Lambayeque-Perú: Universidad Nacional Pedro Ruiz Gallo; 2016. Disponible en: http://repositorio.untumbes.edu.pe/handle/UNITUMBES/78

13. Rodríguez $\mathrm{H}$. Calidad de la Atención en Salud de usuarios internos y externos del servicio de Obstetricia del Hospital Goyenehe de Arequipa - 2015. [Tesis]. Arequipa-Perú: Universidad Católica Santamaría; 2015. Disponible en: http://tesis.ucsm.edu.pe/repositorio/handle/UCS$\mathrm{M} / 5826$ ?show=full

14. Camarena L, Glascoe C. Perspectiva de la calidad de atención prenatal alterna, a la institucional: ciudad de Chihuahua - México. Rev. Fac Nac Salud Pública. 2007; 25(1):40-9. https://www.redalyc.org/ pdf/120/12025106.pdf

15. Munares G, Fausto O. Estudio Sobre Calidad del control prenatal en establecimientos de salud del primer nivel de atención de Lima. [Tesis]. Lima-Perú: Universidad Nacional Mayor de San Marcos; 2005. Disponible en: https://hdl.handle.net/20.500.12672/1771

16. Aguilar I. Medición de factores que influyen en la calidad de atención prenatal por enfermeras en los distritos del área de salud de Totonicapán - 2015. [Tesis]. Quetzaltenango-Guatemala: Universidad Rafel Landívar de Guatemala; 2015. Disponible en: http://recursosbiblio.url.edu .gt/tesiseortiz /2016/09/02/Aguilar-Ingrid.

17. Noguera A, Dueñas M. Evaluación de la Calidad de Atención del Programa de Control Prenatal a partir de la atención brindada a las usuarias del centro de salud suroccidente en la ESE POPAYAN de enero a junio 2011. [Tesis]. Popayán-Colombia. Universidad del Cauca de Colombia; 2011. Disponible en:https://repository.ean.edu.co/bitstream/handle/10882/1734/NogueraAstrid2011.pdf?sequence=1

18. Delgado R, et al. Grado de Satisfacción Laboral del Usuario Interno en los Establecimientos de Salud de la Red Quispicanchi - Cusco. SITUA XXIII Rev. Fac Medicina Humana UNSAAC. 2003; 12(23):36-43. https://sisbib. unmsm.edu.pe/bvrevistas/situa/2004_n23/grado.htm 